\title{
Energy Devices for Sensor Networks: Properties for Simulation and Deployment
}

\author{
Alex S. Weddell, Geoff V. Merrett, Nick R. Harris, and Neil M. White \\ Pervasive Systems Centre, School of Electronics and Computer Science \\ University of Southampton \\ Southampton, UK, SO17 1BJ \\ \{asw,gvm,nrh,nmw\}@ecs.soton.ac.uk
}

\begin{abstract}
Recent developments in microcontroller, radio transceiver, and energy harvesting device design now permit wireless sensor nodes to operate indefinitely from power scavenged from their environment. Many algorithms for conventional sensor networks assume that nodes run directly from non-rechargeable batteries and therefore attempt to conserve energy rather than carefully exploiting it when available. Effectively incorporating energy harvesting into wireless sensor network deployments, and simulations, poses unique problems related to energy-awareness and performance optimization. This paper presents a characterization of energy devices for sensor nodes and outlines their use in simulation and deployment. A case study of an energy-aware sensor node operating from a photovoltaic module and supercapacitor is explored. This paper also presents a modular software and hardware architecture which encourages energy-aware algorithm design and allows the automatic configuration of energy-aware sensor nodes.
\end{abstract}

Keywords-energy harvesting; energy management; modeling; simulation; wireless sensor networks

\section{INTRODUCTION}

Wireless sensor networks are collections of sensor nodes which are deployed to monitor parameters including temperature, pressure, or vibration, in locations such as machines, buildings, or the environment. The wireless sensor nodes considered in this paper typically use low-power microcontrollers such as the Texas Instruments CC2430 [1] and draw relatively small currents (nominally $<1 \mu \mathrm{A}$ when sleeping and $\sim 25 \mathrm{~mA}$ when active and communicating). They can deliver a long operational lifetime from primary batteries by maintaining a low duty cycle; however, recent developments in energy harvesting (scavenging energy from light, vibration, or thermal gradients) means that sensor nodes can potentially operate indefinitely from only environmental energy. The effective use of this harvested energy can pose challenges, as the nodes no longer need to minimize their duty cycle but, instead, optimize their operation with regard to the rate of power generation and amount of stored energy.

A number of recent projects have used energy harvesting, including Prometheus (using a photovoltaic module in combination with a two-stage energy storage system

Manuscript received March 2, 2009. This work was supported in part by the Engineering and Physical Sciences Research Council (EPSRC) under grant number EP/D042917/1. incorporating supercapacitors) [2]. The energy models used by Prometheus are relatively simplistic, with voltage thresholds triggering system operation. Other projects have integrated multiple energy harvesting sources into the energy subsystem of a sensor node (for example AmbiMax [3]) but, again, with only coarse energy-aware algorithms. Energy awareness is important in state-of-the-art routing algorithms; for example Voigt et al. [4] developed a solar-aware routing algorithm. Recent system deployments such as Trio [5] exposed the issue that the actual performance of nodes powered by energy harvesting can differ from their simulation. Our approach is to develop energy models which can be exploited in both deployment (to improve energy-awareness of nodes) and simulation (to improve system design processes). By using models in both simulation and deployment, the correlation of simulation with actual performance may be improved.

This paper looks at representing the energy subsystem of sensor nodes as a sequence of characterized modules. The need for such device models is explored in Section II and the properties of real devices are described in Section III. Novel architectures for the embedded software of the sensor node (used in simulation and deployment) and its hardware interfaces are explored in Section IV.

\section{Why MODEL ENERGY DEVICES?}

Device models provide the building blocks of simulation; indeed a simulation is only as realistic as the models it is built around. To ensure a close correlation between simulation and practical results, accurate models are needed; in order to sufficiently evaluate energy-aware networks, energy device models are paramount.

Device models are also required in a node's embedded firmware in order to deliver energy-awareness. Energyawareness is particularly important in sensor nodes incorporating energy harvesting as it allows nodes to optimize their use of the energy available, rather than simply minimizing their energy usage. To achieve this, it is necessary to interface with energy devices to interrogate them and determine their state. For example, the microcontroller should measure the voltage on an energy store (for example a supercapacitor) in order to determine the stored energy, or the fraction of operating time remaining. Similarly the nominal power from an energy source (for example a photovoltaic 
module) can be estimated by measuring its open-circuit voltage. This permits the node to measure its energy status and use this to decide its participation in the network, its duty cycle, or the amount of energy it wishes to expend performing sensing tasks.

\section{PROPERTIES OF ENERGY DEVICES}

We adopt the approach of classifying energy devices as sources, stores, and consumers [8]. The representation of the energy subsystem of the sensor node can be simplified by classifying components thus:

- Energy Sources: devices that source (or provide) energy; for example photovoltaic modules or vibration harvesters.

- Energy Stores: devices that store (or buffer) energy; for example batteries and supercapacitors.

- Energy Consumers: devices that consume (or use) energy; for example microcontrollers and peripherals.

The operating parameters of devices can be obtained through theoretical modeling, empirical observation, or the use of manufacturers' models. Effectively, these parameters must be sufficient to ascertain the following:

- Power Generated: for energy sources, the rate of power generation under given conditions.

- Energy Stored: for energy stores, how much energy is stored under given conditions.

- Discharge Dynamics: for energy consumers, the dynamics of their energy usage.

The meanings of these parameters in the context of a Texas Instruments CC2430EM [1] wireless sensor node powered by a photovoltaic module and a supercapacitor are considered in the following subsections by way of datasheet parameters and empirical observations of the behavior of individual components of the system.

\section{A. Energy Source: Photovoltaic Module Properties}

The open-circuit voltage of the photovoltaic module in response to changing light levels can be approximated by a logarithmic curve as shown in Fig. 1. The curve shown is for a 90x72mm amorphous silicon device.

$$
P_{\text {nom }}=\left(V_{o c} \cdot k\right)\left(e^{\frac{V_{o c}-B}{A}} \cdot m\right)
$$

The nominal output power is estimated by (1), where $V_{o c}$ is the open-circuit voltage $(\mathrm{V}), k$ is the ratio between $V_{o c}$ and the maximum power point voltage ( 0.71 for this device), $A$ and $B$ are the logarithmic parameters $(0.2571$ and 2.9128 for this device) and $m$ is the ratio between illuminance and maximum power point current $\left(5.4 \times 10^{-7}\right.$ for this device) [6]. This allows the nominal power (which is the power generated at its maximum power point) of the module to be estimated.

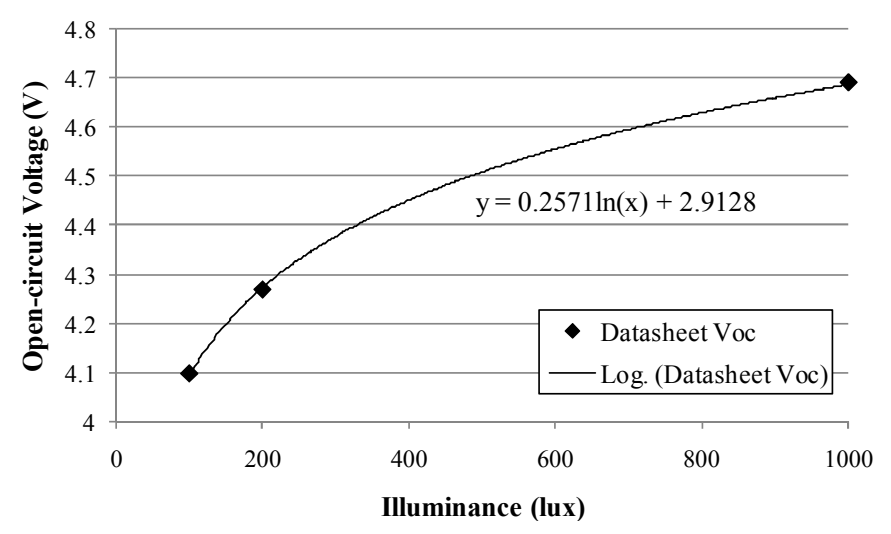

Figure 1. Performance of an amorphous silicon photovoltaic module.

To operate at the maximum power point the cell must be driven across a certain voltage; if connected directly to a supercapacitor this cannot be maintained as the energy stored in the supercapacitor controls the voltage across the cell. Therefore the energy harvested from the cell will often be lower than the nominal power. Further information on maximum power points and cell characteristics can be found in [7].

\section{B. Energy Store: Supercapacitor Properties}

As highlighted in Section 1, supercapacitors are increasingly being used to power wireless sensor nodes; however, their operation differs considerably from that of conventional batteries and consideration must be given to this in both simulations and energy-aware algorithms. The first major difference to note is that the voltage across a supercapacitor varies significantly with the energy stored inside it, following the standard equation for a capacitor, as shown in (2) where $E$ is the stored energy (J), $C$ is the capacitance $(\mathrm{F})$ and $V$ is the voltage across the store $(\mathrm{V})$.

$$
E=\frac{C V^{2}}{2}
$$

Unless additional voltage-conversion hardware is employed on the node, the usable stored energy is considerably lower than the stored energy (as most microcontrollers cease to operate below $2 \mathrm{~V}$ ); for example, only arount $50 \%$ of the energy stored in a $1.8 \mathrm{~F}$ supercapacitor at $2.75 \mathrm{~V}$ is available at a voltage of over $2 \mathrm{~V}$. Furthermore, the rated capacitance of a supercapacitor has a wide tolerance, typically $(-20 \% /+40 \%)$.

Supercapacitors often exhibit considerable internal leakage, and this leakage varies with the stored energy, manufacturer, and individual device [8]. Leakage is of particular relevance to their use as energy stores for wireless sensor nodes, as the supercapacitor could self-discharge to below a usable $2 \mathrm{~V}$ within a matter of hours. The leakage power versus residual energy characteristics can be modeled by approximating empirical results, and this can be used in simulation to predict the behavior of the supercapacitor; for example this can be integrated into a discrete event simulator by using (3) at discrete intervals of time $\Delta t$ (assuming that $E_{r}$ does not change 


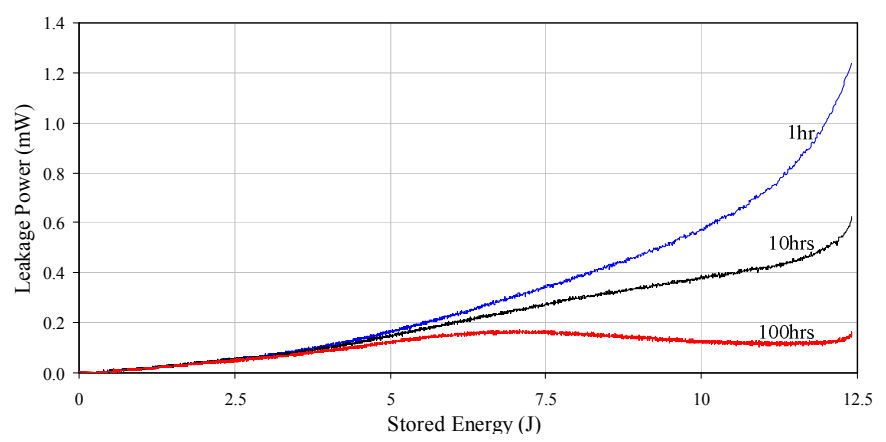

Figure 2. Leakage power exhibited by a $4.7 \mathrm{~F}$ supercapacitor.

significantly over the period $\Delta t$ ), where $E_{l}$ is the energy (J) leaked over period $\Delta t(\mathrm{~s})$, and $P_{l}\left(E_{r}\right)$ is the leakage power (W) for a residual energy of $E_{r}(\mathrm{~J})$.

$$
E_{l}\left(\Delta t, E_{r}\right)=\Delta t \cdot P_{l}\left(E_{r}\right)
$$

As shown in Fig. 2 (where data has been obtained from empirical measurements, operating a $4.7 \mathrm{~F}$ supercapacitor with a maximum operating voltage of $2.3 \mathrm{~V}$ ), the leakage power is also heavily dependent on the period of time that the supercapacitor is 'held' at its maximum operating voltage before discharging. It can be seen that a reduction in the leakage power of an order of magnitude can be obtained by holding the supercapacitor at its maximum operating voltage for 100 hours instead of 1 hour (the reasons for - and modeling of - this effect is currently being investigated further). Therefore, in scenarios where the supercapacitor is likely to cycle regularly (for example in an application using solar energy), the leakage is likely to be higher than in scenarios where the supercapacitor cycles less frequently.

Supercapacitors exhibit complex properties that makes their high-level and 'simplistic' modeling difficult. In order to obtain realistic modeling (and hence simulation), it is important to consider the application into which the supercapacitor will be deployed.

\section{Energy Consumer: CC2430 Microcontroller/Transceiver}

Assessing the energy status of a node driven from a supercapacitor is a non-trivial task, and is dependent on the dynamics of the current draw of the sensor node in response to a changing supply voltage [9].

Investigations with a CC2430EM sensor node on a constant sense/transmit cycle, as shown in Fig. 3, show that the current draw changes little (unlike the power consumption); hence it can be modeled as a current-dominated consumer.

The fraction of operating time remaining (compared to when the store is full), $t_{\text {frac }}$, is equivalent to the energy fraction, $E_{\text {frac }}$, calculated using (4), where $E_{\text {present }}$ is the amount of energy stored at a given time, $E_{\min }$ is the minimum amount of energy required to provide the minimum operating voltage for the node, and $E_{\max }$ is the energy stored at maximum voltage.

$$
t_{\text {frac }}=E_{\text {frac }}=\frac{E_{\text {present }}-E_{\text {min }}}{E_{\text {max }}-E_{\min }}
$$

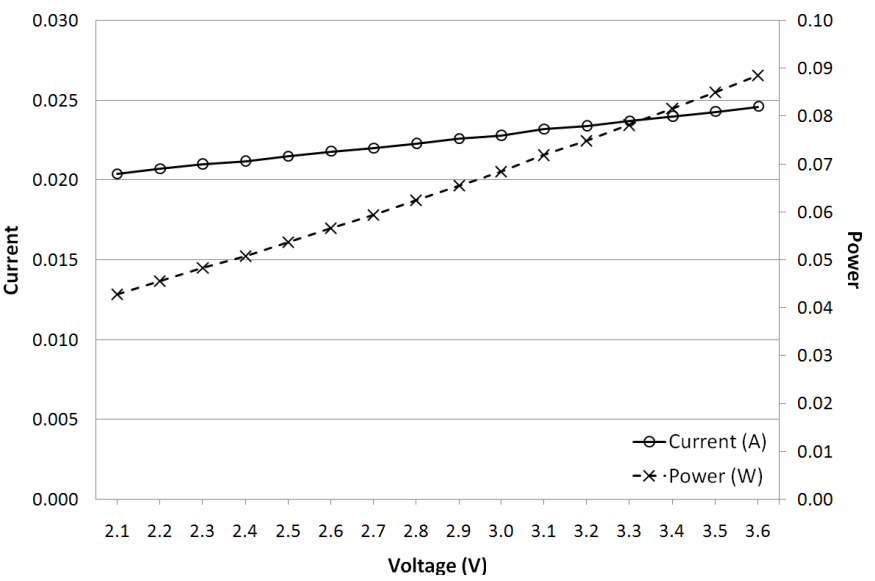

Figure 3. Current and power consumption of CC2430EM under a varying supply voltage.

The fractional operating time is the preferred metric to use to state the energy status of the node as it takes its discharge dynamics into account. Other equations are available for power and resistance dominated discharge types [9].

\section{NOVEl ENERGY-AwARE ARCHITECTURES}

To use device models during the operation of the node, we have devised novel hardware and software architectures. The hardware architecture facilitates the connection of a variety of energy modules to the system and allows the operational parameters of devices to be stored on-module, which provides a means for the microcontroller to interrogate this 'datasheet' and to obtain parameters for, and manage, each device individually. A novel software architecture has also been developed, which is configured to interface with the new hardware architecture and provides the application running on the sensor node with a modularized method for managing its energy subsystem.

\section{A. Hardware Interfaces}

A simple way of making nodes energy-aware is to hardwire connections between the microcontroller and its energy devices, and to include their operating parameters in the embedded software of the sensor node; however, this is a relatively inflexible arrangement as it does not support the changing of energy hardware after the node has been programmed (for example, when the selection of energy stores and sources is carried out at the time of deployment, when the properties of the environment are clearer). Rather, in our developed system we introduce a novel concept of the 'energy electronic datasheet' (EEDS), which is a development from the 'component electronic datasheet', proposed by Bandari et al. [10]. This permits the operational parameters of each energy device to be stored in a local EPROM, which is interrogated by the microcontroller when the device is connected to the system or when the system is first activated.

A hardware interface has been produced which permits the sensor node to interface with its energy hardware, facilitating the connection of up to six energy devices (including photovoltaics and supercapacitors) to the system through an 'energy multiplexer' module. This permits the sensor node to 
query the EEDS on energy devices to obtain their operating parameters, and interrogate and manage individual devices.

For example, the datasheet on the photovoltaic module stores the parameters stated in Section III.A of this paper, and the interface also permits the node to disconnect the photovoltaic device from its load in order to measure its $V_{o c}$ and hence calculate the nominal power. The prototype system is shown in Fig. 4, and incorporates a number of devices, including the supercapacitor and photovoltaic devices and CC2430 microcontroller. The 10-wire connection between the microcontroller and the 'multiplexer module' is shown in Table I and the 8-wire connection between the 'multiplexer module' and the other energy modules is shown in Table II.

The default operation of the system is to obtain energy from the energy harvesting sources and to store unused energy in the supercapacitor energy store. The pictured prototype also includes modules incorporating a primary battery and a mains adapter, which means that any form of electrical energy source can be exploited, when available, through the connection of additional modules to the system.

\section{B. Software Architectures}

The system makes use of a novel energy-aware software architecture shown in Fig. 5. This architecture differs from conventional software structures in that the energy management tasks are split into a separate stack rather than being combined in the communication stack's application layer. This brings a number of benefits including modularization and improving the readability of code and the reusability of layers [11].

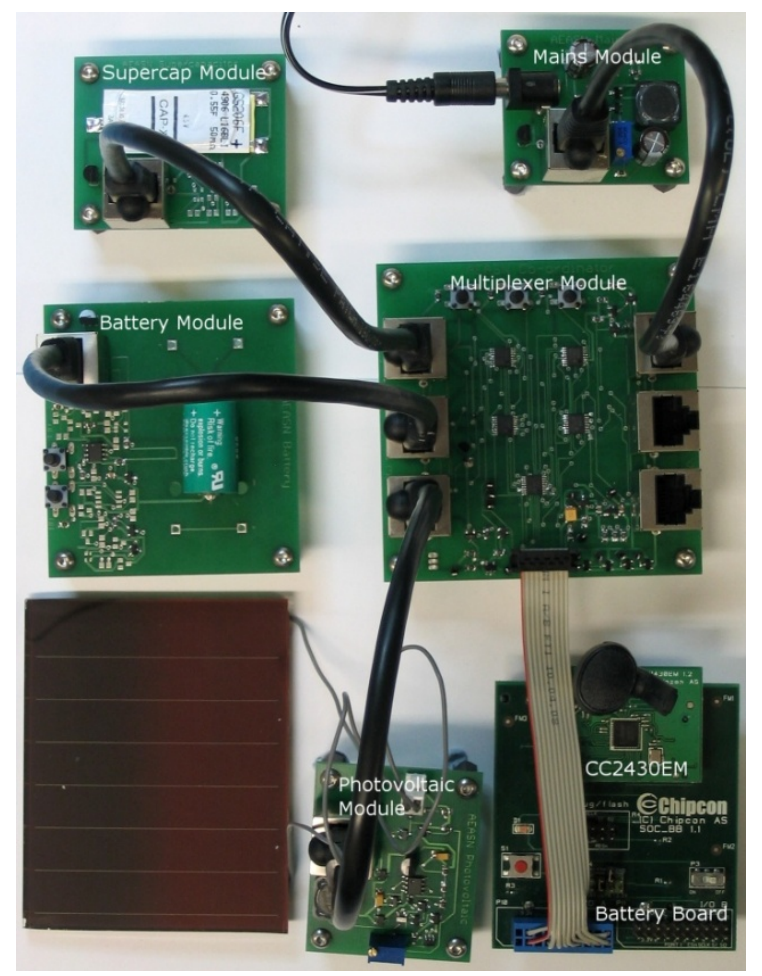

Figure 4. Prototype EEDS system incorporating a number of energy modules.
TABLE I CONNECTIONS BETWEEN MICROCONTROLLER AND MULTIPLEXER MODULE

\begin{tabular}{|c|l|l|}
\hline Pin & Connector & Description \\
\hline 1 & Mux Address 0 & \\
2 & Mux Address 1 & Digital lines to control all multiplexers \\
3 & Mux Address 2 & \\
\hline 4 & 1-wire EEDS line & Digital 1-wire interface \\
\hline 5 & Bi-dir Recon A & \\
6 & Bi-dir Recon B & Lines to reconfigurable I/O pins on $\mu \mathrm{C}$ \\
7 & Bi-dir Recon C & (capable of both digital I/O and ADC) \\
8 & Bi-dir Recon D & \\
\hline 9 & Vreg & Supply voltage output (direct to node) \\
10 & GND & \\
\hline
\end{tabular}

TABLE II. CONNECTIONS BETWEen MultiPLEXeR MOdULE AND ENERGY MODULES

\begin{tabular}{|c|l|l|}
\hline Pin & Connector & Description \\
\hline 1 & 1-wire EEDS line & Digital 1-wire EEDS interface \\
\hline 2 & Bi-dir Recon A & \\
3 & Bi-dir Recon B & Lines to reconfigurable I/O pins on $\mu \mathrm{C}$ \\
4 & Bi-dir Recon C & Directed via multiplexers \\
5 & Bi-dir Recon D & \\
\hline 6 & Vreg & Regulated voltage used as supply to $\mu \mathrm{C}$ \\
\hline 7 & Vout & Voltage interface \\
8 & GND & \\
\hline
\end{tabular}

The energy stack manages the interface with energy devices, with the following tasks taking place at each layer:

- Physical Energy: interfaces with the microcontroller ADCs in order to obtain raw voltage readings; sets appropriate outputs.

- Energy Analysis: analyses data from the physical energy layer, translating voltages into estimates of stored energy or generated power.

- Energy Control: provides an interface to the application running on the sensor node, classifying the overall energy status of the node and allowing the application to query energy levels.

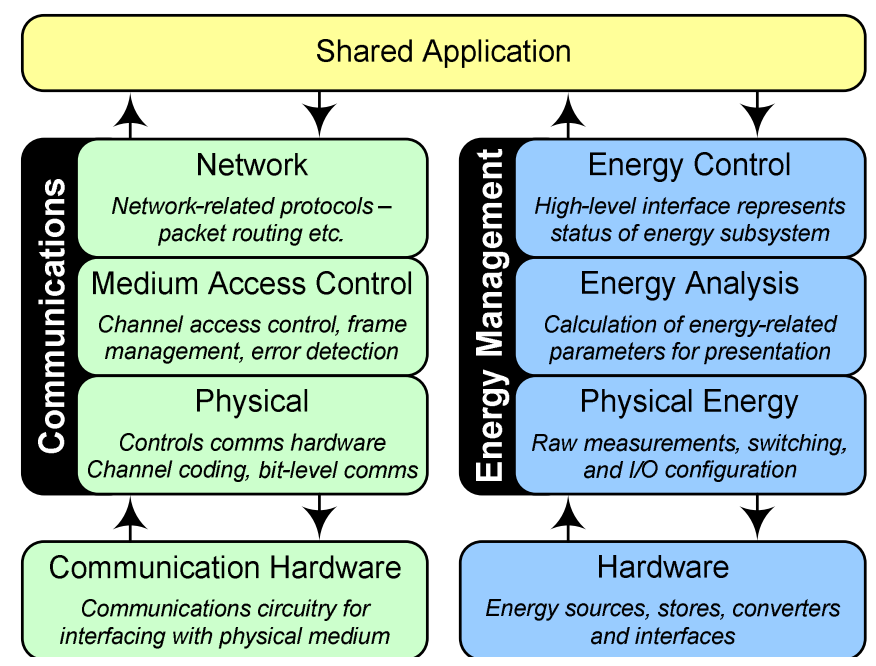

Figure 5. The multi-stack architecture used in the embedded software of the developed prototype system. 
In this prototype system, the physical energy layer performs switching of the outputs to select the correct multiplexer channel, to permit the interrogation of the photovoltaic device, and to obtain raw ADC readings related to the supercapacitor voltage and the $V_{o c}$ of the photovoltaic device. The energy analysis layer uses the device models (with the equations presented earlier) in combination with the device parameters obtained from their EEDSs, along with the sensed values, in order to estimate their power and energy. This is then used by the energy control layer to classify the power status of the system into 'power priorities' which are then used by the application to determine the participation of the sensor node in the network.

\section{CONCLUSIONS}

In this paper we have outlined how device models can be incorporated into deployments of wireless sensor nodes to achieve energy-aware operation and used in simulation to achieve accurate results. Particular examples of supercapacitor and photovoltaic cells have been described. The proposed hardware/software architecture incorporates a software structure which devolves energy management into its own stack, and a new hardware structure which allows a number of energy modules to be connected to the system.

We have shown that accurate device models are relevant to sensor nodes with complex energy resources. Our system incorporates a hardware architecture that allows energy devices to be exchanged, with the node's embedded software reacting accordingly. This is a realization of a new process where device models are used in both simulation and deployment to achieve better levels of energy-awareness and hence improve the exploitation of energy resources.

\section{REFERENCES}

[1] Texas Instruments, "System-on-Chip Solution for $2.4 \mathrm{GHz}$ IEEE 802.15.4/ZigBee ${ }^{\mathrm{TM}}$," [online] available http://www.ti.com/cc2430, last accessed March 2009.

[2] X. Jiang, J. Polastre, and D. Culler, D., "Perpetual environmentally powered sensor networks," in Proc. $4^{\text {th }}$ Int'l Symp. Information Processing in Sensor Networks, 2005.

[3] C. Park and P.H. Chou, "AmbiMax: autonomous energy harvesting platform for multi-supply wireless sensor nodes," in Proc. $3^{\text {rd }}$ IEEE Communications Society Conf. Sensor and Ad Hoc Comms and Networks, 2006.

[4] T. Voigt, H. Ritter, J. Schiller, "Utilizing solar power in wireless sensor networks," in Proc. 28 th IEEE Int'l Conf. Local Computer Networks, 2003.

[5] P. Dutta et al., "Trio: enabling sustainable and scalable outdoor wireless sensor network deployments," in Proc. $5^{\text {th }}$ Int'l Conf. Information Processing in Sensor Networks, 2006.

[6] A.S. Weddell, N.R. Harris, and N.M. White, "An Efficient Indoor Photovoltaic Power Harvesting System for Energy-Aware Wireless Sensor Nodes," in Proc. Eurosensors, 2008.

[7] D. Dondi, D. Brunelli, L. Benini, P. Pavan, A. Bertacchini, and L. Larcher, "Photovoltaic cell modeling for solar energy powered sensor networks," in Proc. Int'l Workshop Advances in Sensors and Interface, 2007.

[8] G.V. Merrett, A.S.Weddell, A.P. Lewis, N.R. Harris, B.M. Al-Hashimi, and N.M. White, "An Empirical Energy Model for Supercapacitor Powered Wireless Sensor Nodes," Proc. 17 $7^{\text {th }}$ Int'l Conf. Computer Communications and Networks (ICCCN), 2008.

[9] A.S. Weddell, N.R. Harris, and N.M. White, "Alternative Energy Sources for Sensor Nodes: Rationalized Design for Long-Term Deployment," in Proc. Int'l Instrumentation and Measurement Technology Conference, 2008.

[10] S. Bandari, C. Santiago, H.S. Mohammed, and J. Schmalzel, "Component electronic datasheets in ISHM," in Proc. IEEE Sensors Applications Symposium, 2006.

[11] G.V. Merrett, A.S. Weddell, N.R. Harris, and N.M. White, "A Structured Hardware/Software Architecture for Embedded Sensor Nodes," in Proc. 17 $7^{\text {th }}$ Int'l Conf. Computer Communications and Networks, 2008. 